\title{
Some new families of Tasoevian and Hurwitzian continued fractions
}

by

\author{
JAMes MC LAughlin (West Chester, PA)
}

1. Introduction. In this paper we exhibit several new infinite families of regular continued fractions of Hurwitzian and Tasoevian type, continued fractions whose value can be expressed in terms of certain infinite series.

Hurwitzian continued fractions ([2], [3]) are of the form

$$
\begin{aligned}
{\left[a_{0} ; a_{1}, \ldots, a_{k}, f_{1}(1), \ldots, f_{n}(1)\right.} & \left., f_{1}(2), \ldots, f_{n}(2), \ldots\right] \\
= & :\left[a_{0} ; a_{1}, \ldots, a_{k}, \overline{f_{1}(m), \ldots, f_{n}(m)}\right]_{m=1}^{\infty} .
\end{aligned}
$$

Here the $f_{i}(x)$ are polynomials with rational coefficients taking only positive integral values for integral $x \geq 1$, and at least one is non-constant. The integer $n$ is termed the quasi-period of the continued fraction. The closed form for Hurwitzian continued fractions is not known in general. This class contains numbers like

$$
\begin{aligned}
e & =[2 ; 1,2,1,1,4,1,1,6,1, \ldots]=[2 ; \overline{1,2 m, 1}]_{m=1}^{\infty}, \\
\tan 1 & =[1 ; 1,1,3,1,5,1,7,1,9, \ldots]=[1 ; \overline{2 m-1,1}]_{m=1}^{\infty} .
\end{aligned}
$$

These continued fractions were also investigated by D. N. Lehmer [13], D. H. Lehmer [14], and more recently by Komatsu in [5], [7], [8], [10] and [11]. A nice example that follows from Lambert's [12] continued fraction

$$
\frac{e^{z}-e^{-z}}{e^{z}-e^{-z}}=\frac{z}{1}+\frac{z^{2}}{3}+\frac{z^{2}}{5}+\frac{z^{2}}{7}+\cdots
$$

is the following (see also [5]):

$$
\sqrt{\frac{v}{u}} \tan \frac{1}{\sqrt{u v}}=[0 ; u-1, \overline{1,(4 k-1) v-2,1,(4 k+1) u-2}]_{k=1}^{\infty} .
$$

2000 Mathematics Subject Classification: Primary 11A55.

Key words and phrases: continued fractions, Tasoevian continued fractions, Hurwitzian continued fractions. 
A subclass of Hurwitzian continued fractions (with all polynomials $f_{i}(x)$ of degree 1) is due to D. H. Lehmer [14], who found closed forms for the numbers represented by regular continued fractions whose partial quotients were terms in an arithmetic progression,

$$
[0 ; a, a+b, a+2 b, a+3 b, \ldots]=\frac{I_{(a / b)}(2 / b)}{I_{(a / b)-1}(2 / b)},
$$

where

$$
I_{\nu}(z)=\sum_{m=0}^{\infty} \frac{(z / 2)^{\nu+2 m}}{\Gamma(m+1) \Gamma(\nu+m+1)} .
$$

More transparently,

$$
[0 ; a, a+b, a+2 b, a+3 b, \ldots]=\frac{1}{b} \frac{\sum_{k=0}^{\infty} \frac{b^{-2 k}}{(a / b)_{k+1} k !}}{\sum_{k=0}^{\infty} \frac{b^{-2 k}}{(a / b)_{k} k !}}
$$

where $(a / b)_{0}=1$ and $(a / b)_{k}=(a / b)(a / b+1) \cdots(a / b+k-1)$ for $k>0$.

Lehmer also evaluated continued fractions whose partial quotients consisted of two interlaced arithmetic progressions. Let $a, b, c$ and $d$ be integers satisfying

$$
2 b c=d(2 a+b)
$$

Then

$$
[0 ; a, c, a+b, c+d, a+2 b, c+2 d, \ldots]=\sqrt{\frac{d}{b}} \frac{I_{(2 a / b)}(4 / \sqrt{b d})}{I_{(2 a / b)-1}(4 / \sqrt{b d})} .
$$

An example that Lehmer gave of the former type was the following:

$$
[1 ; 2,3,4,5, \ldots]=\frac{\sum_{m=0}^{\infty} \frac{1}{(m !)^{2}}}{\sum_{m=0}^{\infty} \frac{1}{m !(m+1) !}}
$$

Tasoev [18], [19] proposed a new type of continued fraction of the form $(1.3)$

$$
[a_{0} ; \underbrace{a, \ldots, a}_{m}, \underbrace{a^{2}, \ldots, a^{2}}_{m}, \underbrace{a^{3}, \ldots, a^{3}}_{m}, \ldots]
$$

where $a_{0} \geq 0, a \geq 2$ and $m \geq 1$ are integers. This type was further investigated by Komatsu in [4], where he derived a closed form for the general case ( $m \geq 1$, arbitrary). Komatsu gave several variations of Tasoevian continued fractions in [5]-[8]. In [16], the present author and Nancy Wyshinski derived several variations of Tasoev's continued fraction from known results about 
$q$-continued fractions. Two examples of our results from that paper are the following.

Example 1. Define

$$
F(c, d, q):=\sum_{n=0}^{\infty} \frac{(-1)^{n} c^{n} q^{n(n+1) / 2}}{(q ; q)_{n}(c q / d ; q)_{n}}
$$

and let $\omega=e^{2 \pi i / 3}$. If $a>1$ is an integer and $c$ is a rational such that $a / c$ is an integer, $a / c>2$, then

$$
\left[0 ; \frac{a}{c}-2, \overline{1, \frac{a^{k+1}}{c}-3}\right]_{k=1}^{\infty}=\frac{(c / a) F\left(-c \omega / a, \omega^{2}, 1 / a\right)}{\left(1+c \omega^{2} / a\right) F\left(-c \omega, \omega^{2}, 1 / a\right)} .
$$

Example 2. For $r, s$ and $q \in \mathbb{C}$ with $|q|<1$, define

$$
\phi(r, s, q)=\sum_{n=0}^{\infty} \frac{q^{\left(n^{2}+n\right) / 2} r^{n}}{(q ; q)_{n}(-s q ; q)_{n}} .
$$

Let $m$ and $n$ be positive integers and let $d$ be rational such that $d n \in \mathbb{Z}^{+}$ and $d m n>1$. If $n>2$ and $m>1$ then

$$
\begin{aligned}
{\left[0, \overline{1, d^{2 k-2} n^{2 k-1}-2,1, m^{2 k-1}-1, d^{2 k-1} n^{2 k}, m^{2 k}-1}\right]_{k=1}^{\infty} } & \\
& =1+\frac{\phi(d m, d,-1 / d m n)}{\phi(-1 / n, d,-1 / d m n)} .
\end{aligned}
$$

In the present paper we continue our work with $q$-continued fractions, giving $q$-continued fraction proofs for some existing families of Tasoevian and Hurwitzian continued fractions. In addition, we also find the limits of some new families of Tasoevian and Hurwitzian continued fractions.

We also evaluate various finite continued fractions containing arithmetic progressions, deriving Lehmer's results in the limit.

2. Tasoevian continued fractions. In [1], the following result on $q$ continued fractions was proved:

TheOrem 1. Let $a, b, c, d$ be complex numbers with $d \neq 0$ and $|q|<1$. Define

$$
H_{1}(a, b, c, d, q):=\frac{1}{1}+\frac{-a b q+c}{(a+b) q+d}+\cdots+\frac{-a b q^{2 n+1}+c q^{n}}{(a+b) q^{n+1}+d}+\cdots .
$$

Then

$$
\frac{1}{H_{1}(a, b, c, d, q)}-1=\frac{c-a b q}{(d+a q) q} \frac{\sum_{j=0}^{\infty} \frac{(b / d)^{j}(-c / b d)_{j} q^{(j+1)(j+2) / 2}}{(q)_{j}\left(-a q^{2} / d\right)_{j}}}{\sum_{j=0}^{\infty} \frac{(b / d)^{j}(-c / b d)_{j} q^{j(j+1) / 2}}{(q)_{j}(-a q / d)_{j}}}
$$


Here we are employing the standard notation for $q$-products:

$$
(z)_{0}:=(z ; q)_{0}:=1, \quad(z)_{n}:=(z ; q)_{n}:=\prod_{k=0}^{n-1}\left(1-z q^{k}\right) \quad \text { if } n \geq 1 .
$$

This theorem immediately leads to some general results concerning Tasoevian continued fractions.

Theorem 2. Let $c$, e and $m$ be integers, $m>1$, and let $d$ be a rational such that $d m^{2}, d c m / e \in \mathbb{N}$, and $c+d c m / e, e+d m^{2}>0$. Let

$$
a=\frac{e-\sqrt{e^{2}+4 e / c}}{2}, \quad b=\frac{e+\sqrt{e^{2}+4 e / c}}{2} .
$$

Then

$$
\begin{aligned}
{\left[0 ; \overline{c+\frac{d c}{e} m^{2 n-1}, e+d m^{2 n}}\right]_{n=1}^{\infty} } \\
=\frac{e / c}{m d+a} \frac{\sum_{n=0}^{\infty} \frac{(b / d)^{n} m^{-n(n+3) / 2}}{(1 / m ; 1 / m)_{n}\left(-a / d m^{2} ; 1 / m\right)_{n}}}{\sum_{n=0}^{\infty} \frac{(b / d)^{n} m^{-n(n+1) / 2}}{(1 / m ; 1 / m)_{n}(-a / d m ; 1 / m)_{n}}}
\end{aligned}
$$

Proof. With the stated values of $a$ and $b$,

$$
\begin{aligned}
& {\left[0 ; \overline{c+\frac{d c}{e} m^{2 n-1}, e+d m^{2 n}}\right]_{n=1}^{\infty}} \\
& =\frac{1}{-((a+b)+d m) /(a b)}+\frac{1}{(a+b)+d m^{2}}+ \\
& \cdots+\frac{1}{-\left((a+b)+d m^{2 n-1}\right) /(a b)}+\frac{1}{(a+b)+d m^{2 n}}+\cdots \\
& =\frac{-a b}{(a+b)+d m}+\frac{-a b}{(a+b)+d m^{2}}+\cdots+\frac{-a b}{(a+b)+d m^{n}}+\cdots \\
& =\frac{-a b / m}{(a+b) / m+d}+\frac{-a b / m^{3}}{(a+b) / m^{2}+d}+\cdots+\frac{-a b / m^{2 n-1}}{(a+b) / m^{n}+d}+\cdots \text {. }
\end{aligned}
$$

The result now follows from (2.1), upon setting $q=1 / m$ and $c=0$.

Corollary 1. Let $c$ and $m$ be integers, $m>1$, and let $d$ be a positive rational such that $d m \in \mathbb{N}$ and $c+d m>0$. Let

$$
a=\frac{c-\sqrt{c^{2}+4}}{2}, \quad b=\frac{c+\sqrt{c^{2}+4}}{2} .
$$


Then

$$
\left[0 ; \overline{c+d m^{n}}\right]_{n=1}^{\infty}=\frac{1}{m d+a} \frac{\sum_{n=0}^{\infty} \frac{(b / d)^{n} m^{-n(n+3) / 2}}{\sum_{n=0}^{\infty} \frac{(b / d)^{n} m^{-n(n+1) / 2}}{(1 / m ; 1 / m)_{n}\left(-a / d m^{2} ; 1 / m\right)_{n}}}}{(1 / m ; / m)_{n}(-a / d m ; 1 / m)_{n}}
$$

Proof. Let $e=c$ in Theorem 2.

REmarks. (1) We believe that the limit of the general Tasoevian continued fraction of the form $\left[0 ; \overline{c+d m^{n}}\right]_{n=1}^{\infty}$ has not been evaluated before, although special cases have occurred in the literature, such as $\left[0 ; \overline{d m^{n}}\right]_{n=1}^{\infty}$ by Komatsu in [5]. We believe that the evaluation of the general Tasoevian continued fraction $\left[0 ; \overline{c+\frac{d c}{e} m^{2 n-1}, e+d m^{2 n}}\right]_{n=1}^{\infty}$ is also new.

(2) It is clear from Theorem 1 that (2.3) also holds for many cases where the partial quotients in (2.2) or (2.3) are not positive integers. In particular, we can let the parameters assume negative values and then convert the resulting continued fractions to regular continued fractions by removing any resulting non-positive partial quotients. This will produce still further general classes of Tasoevian continued fractions.

To accomplish this, we recall, as noted in [17], that

$$
[m, n, 0, p, \alpha]=[m, n+p, \alpha] \quad \text { and } \quad[m,-n, \alpha]=[m-1,1, n-1,-\alpha] .
$$

We give two examples to illustrate the phenomenon, using the continued fraction at (2.2):

Corollary 2. Let $c$, e and $m$ be integers, $m>1$, and let $d$ be a positive rational such that $d m^{2}, d c m / e \in \mathbb{N}$. Let

$$
a=\frac{e-\sqrt{e^{2}+4 e / c}}{2}, \quad b=\frac{e+\sqrt{e^{2}+4 e / c}}{2} .
$$

(i) Suppose that dcm/e-c-2>0 and $d m^{2}+e-2>0$. Then

$$
\begin{aligned}
& {\left[0 ; 1, \frac{d c}{e} m^{2 n-1}-c-2,1, d m^{2 n}+e-2\right]_{n=1}^{\infty}} \\
& \quad=1+\frac{e / c}{-m d+a} \frac{\sum_{n=0}^{\infty} \frac{(b / d)^{n}(-m)^{-n(n+3) / 2}}{(-1 / m ;-1 / m)_{n}\left(-a / d m^{2} ;-1 / m\right)_{n}}}{\sum_{n=0}^{\infty} \frac{(b / d)^{n}(-m)^{-n(n+1) / 2}}{(-1 / m ;-1 / m)_{n}(a / d m ;-1 / m)_{n}}}
\end{aligned}
$$


(ii) Suppose that $d c m / e+c-1>0$ and $d m^{2}-e-2>0$. Then

$$
\begin{aligned}
{\left[0 ; \frac{d c}{e} m+c-1, \overline{1, d m^{2 n}-e-2,1, \frac{d c}{e} m^{2 n+1}+c-2}\right]_{n=1}^{\infty} } \\
=\frac{e / c}{m d+a} \frac{\sum_{n=0}^{\infty} \frac{(-b / d)^{n}(-m)^{-n(n+3) / 2}}{(-1 / m ;-1 / m)_{n}\left(a / d m^{2} ;-1 / m\right)_{n}}}{\sum_{n=0}^{\infty} \frac{(-b / d)^{n}(-m)^{-n(n+1) / 2}}{(-1 / m ;-1 / m)_{n}(-a / d m ;-1 / m)_{n}}} .
\end{aligned}
$$

Proof. The identity at (2.4) follows from (2.2) upon replacing $m$ by $-m$, removing the negative partial quotients from the continued fraction as described above, and finally moving the initial -1 to the right side. The identity at (2.5) follows similarly, upon replacing $d$ by $-d$ and $m$ by $-m$.

Before coming to the next result, we need some more terminology. We call $d_{0}+K_{n=1}^{\infty} c_{n} / d_{n}$ a canonical contraction of $b_{0}+K_{n=1}^{\infty} a_{n} / b_{n}$ if

$$
C_{k}=A_{n_{k}}, \quad D_{k}=B_{n_{k}} \quad \text { for } k=0,1,2, \ldots,
$$

where $C_{n}, D_{n}, A_{n}$ and $B_{n}$ are canonical numerators and denominators of $d_{0}+K_{n=1}^{\infty} c_{n} / d_{n}$ and $b_{0}+K_{n=1}^{\infty} a_{n} / b_{n}$ respectively. From [15, p. 83] we have the following theorem:

THEOREM 3. The canonical contraction of $b_{0}+K_{n=1}^{\infty} a_{n} / b_{n}$ with

$$
C_{k}=A_{2 k}, \quad D_{k}=B_{2 k} \quad \text { for } k=0,1,2, \ldots,
$$

exists if and only if $b_{2 k} \neq 0$ for $k=1,2, \ldots$, and is in this case given by

$$
b_{0}+\frac{b_{2} a_{1}}{b_{2} b_{1}+a_{2}}-\frac{a_{2} a_{3} b_{4} / b_{2}}{a_{4}+b_{3} b_{4}+a_{3} b_{4} / b_{2}}-\frac{a_{4} a_{5} b_{6} / b_{4}}{a_{6}+b_{5} b_{6}+a_{5} b_{6} / b_{4}}+\cdots
$$

The continued fraction (2.7) is called the even part of $b_{0}+K_{n=1}^{\infty} a_{n} / b_{n}$. If a continued fraction converges then of course its even part converges to the same limit.

THEOREM 4. Let $u$ and $v$ be positive integers, $u, v>1$, and let $e$ and $f$ be rationals such that $e u, f v \in \mathbb{N}$. Then

$$
\begin{aligned}
& {\left[0 ; \overline{e u^{n}, f v^{n}}\right]_{n=1}^{\infty}} \\
& \quad=\left(\frac{1}{e u}-\frac{1}{e^{2} f u^{2} v+e}\right) \frac{\sum_{n=0}^{\infty} \frac{(e f)^{-n}(u v)^{-n(n+3) / 2}}{\sum_{n=0}^{\infty} \frac{(e f)^{-n}(u v)^{-n(n+1) / 2}}{(1 / u v ; 1 / u v)_{n}\left(-1 / e f u^{3} v^{2} ; 1 / u v\right)_{n}}}}{(1 / u v ; 1 / u v)_{n}\left(-1 / e f u^{2} v ; 1 / u v\right)_{n}}
\end{aligned}
$$

Proof. We consider the continued fraction

$$
\left[0 ; b_{1}, \overline{e u^{n}, f v^{n}}\right]_{n=1}^{\infty},
$$


with $b_{1}$ an arbitrary positive integer. Clearly, this continued fraction converges, and is thus equal to its even part. By (2.7) this equals

$$
\begin{aligned}
& \frac{e u}{e u b_{1}+1}-\frac{u}{1+(e f u)(u v)+u}-\frac{u}{1+(e f u)(u v)^{2}+u}-\cdots \\
= & \frac{e u}{e u b_{1}+1}-\frac{u /(u v)}{(1+u) /(u v)+e f u}-\frac{u /(u v)^{3}}{(1+u) /(u v)^{2}+e f u}-\cdots .
\end{aligned}
$$

We now apply Theorem 1 to the first tail of the continued fraction above, setting $q=1 / u v, c=0, d=e f u, a=1$ and $b=u$. The result follows upon inverting both the expressions resulting from (2.10) and the continued fraction at (2.9), and then cancelling $b_{1}$.

Remark. Komatsu [5] has a result concerning Tasoevian continued fractions of the form $\left[0 ; \overline{u a^{k}, v b^{k}}\right]_{k=1}^{\infty}$, but he does not explicitly compute the limits, expressing them instead as ratios of series containing certain functions, $R_{0, n}$ and $R_{1, n}$, which are defined recursively for $n \geq 0$. We believe the result in Theorem 4 to be new.

In [14], where Lehmer investigated continued fractions whose partial quotients were in arithmetical progressions, he remarked that it was also possible to evaluate continued fractions in which the terms forming the arithmetic progressions were separated by constant strings of arbitrary partial quotients. We next show that this can also be done with some classes of Tasoevian continued fractions.

TheOREM 5. Let $c$, e and $m$ be integers, with $m>1$. Let $a_{1}, \ldots, a_{k}$ be fixed positive integers and, for $1 \leq i \leq k$, define $P_{i}$ and $Q_{i}$ by

$$
\frac{P_{i}}{Q_{i}}=\frac{1}{a_{1}}+\frac{1}{a_{2}}+\cdots+\frac{1}{a_{i}}
$$

and set $C=Q_{k-1}+P_{k}+c Q_{k}$ and $E=Q_{k-1}+P_{k}+e Q_{k}$. We suppose further that $d$ is a positive rational such that $C d m / E, d m \in \mathbb{N}$.

If $k$ is even, set

$$
a=\frac{E-\sqrt{E^{2}+4 E / C}}{2}, \quad b=\frac{E+\sqrt{E^{2}+4 E / C}}{2} .
$$

Then

$$
\begin{aligned}
& {\left[0 ; \overline{a_{1}, \ldots, a_{k}, c+\frac{C}{E} d m^{2 n-1}, a_{1}, \ldots, a_{k}, e+d m^{2 n}}\right]_{n=1}^{\infty}} \\
& =\frac{P_{k}}{Q_{k}}+\frac{E / C}{Q_{k}\left(m d Q_{k}+a\right)} \frac{\sum_{n=0}^{\infty} \frac{\left(b / d Q_{k}\right)^{n} m^{-n(n+3) / 2}}{\sum_{n=0}^{\infty} \frac{\left(b / d Q_{k}\right)^{n} m^{-n(n+1) / 2}}{(1 / m ; 1 / m)_{n}\left(-a / d Q_{k} m ; 1 / m\right)_{n}}}}{(1 / m)_{n}\left(-a / d Q_{k} m^{2} ; 1 / m\right)_{n}}
\end{aligned}
$$


If $k$ is odd, set

$$
a=\frac{E-\sqrt{E^{2}-4 E / C}}{2}, \quad b=\frac{E+\sqrt{E^{2}-4 E / C}}{2} .
$$

Then

$$
\begin{aligned}
& {\left[0 ; \overline{a_{1}, \ldots, a_{k}, c+\frac{C}{E} d m^{2 n-1}, a_{1}, \ldots, a_{k}, e+d m^{2 n}}\right]_{n=1}^{\infty}} \\
& =\frac{P_{k}}{Q_{k}}+\frac{-E / C}{Q_{k}\left(m d Q_{k}+a\right)} \frac{\sum_{n=0}^{\infty} \frac{\left(b / d Q_{k}\right)^{n} m^{-n(n+3) / 2}}{\sum_{n=0}^{\infty} \frac{\left(b / d Q_{k}\right)^{n} m^{-n(n+1) / 2}}{(1 / m ; 1 / m)_{n}\left(-a / d Q_{k} m ; 1 / m\right)_{n}}}}{(1 / m)_{n}\left(-a / d Q_{k} m^{2} ; 1 / m\right)_{n}}
\end{aligned}
$$

Proof. For any $\alpha$,

$$
\begin{aligned}
{\left[0 ; a_{1}, \ldots, a_{k}, \alpha\right] } & =\frac{\alpha P_{k}+P_{k-1}}{\alpha Q_{k}+Q_{k-1}}=\frac{P_{k}}{Q_{k}}+\frac{\left(P_{k-1} Q_{k}-Q_{k-1} P_{k}\right) / Q_{k}^{2}}{Q_{k-1} / Q_{k}+\alpha} \\
& =\frac{P_{k}}{Q_{k}}+\frac{(-1)^{k} / Q_{k}^{2}}{Q_{k-1} / Q_{k}+\alpha},
\end{aligned}
$$

where the last equality follows from a standard identity in continued fractions. Thus

$$
\begin{aligned}
& {\left[0 ; a_{1}, \ldots, a_{k}, c+\frac{C}{E} d m^{2 n-1}, a_{1}, \ldots, a_{k}, e+d m^{2 n}\right]_{n=1}^{\infty}} \\
& \quad=\frac{P_{k}}{Q_{k}}+\frac{(-1)^{k} / Q_{k}^{2}}{\frac{P_{k}+Q_{k-1}}{Q_{k}}+c+\frac{d C m}{E}+\frac{(-1)^{k} / Q_{k}^{2}}{\frac{P_{k}+Q_{k-1}}{Q_{k}}+e+d m^{2}}+\cdots} \\
& \quad=\frac{P_{k}}{Q_{k}}+\frac{1}{Q_{k}}\left(\frac{(-1)^{k}}{C+\frac{C d Q_{k}}{E} m}+\frac{(-1)^{k}}{E+d Q_{k} m^{2}}+\cdots\right) .
\end{aligned}
$$

If $k$ is even, then

$$
\begin{aligned}
& {\left[0 ; \overline{a_{1}, \ldots, a_{k}, c+\frac{C}{E} d m^{2 n-1}, a_{1}, \ldots, a_{k}, e+d m^{2 n}}\right]_{n=1}^{\infty}} \\
& =\frac{P_{k}}{Q_{k}}+\frac{1}{Q_{k}}\left[0 ; \overline{C+\frac{C d Q_{k}}{E} m^{2 n-1}, E+d Q_{k} m^{2 n}}\right]_{n=1}^{\infty},
\end{aligned}
$$

and (2.11) now follows from Theorem 2. 
If $k$ is odd, then

$$
\begin{aligned}
& {\left[0 ; \overline{a_{1}, \ldots, a_{k}, c+\frac{C}{E} d m^{2 n-1}, a_{1}, \ldots, a_{k}, e+d m^{2 n}}\right]_{n=1}^{\infty}} \\
& =\frac{P_{k}}{Q_{k}}+\frac{1}{Q_{k}}\left[0 ; \overline{(-C)+\frac{(-C) d Q_{k}}{E} m^{2 n-1}, E+d Q_{k} m^{2 n}}\right]_{n=1}^{\infty}
\end{aligned}
$$

and (2.12) likewise follows from Theorem 2 .

COROllary 3. Let $c$ and $m$ be integers, $m>1$, and let $d$ be a positive rational such that $d m \in \mathbb{N}$ and $c+d m>0$. Let $a_{1}, \ldots, a_{k}$ be fixed positive integers and, for $1 \leq i \leq k$, define $P_{i}, Q_{i}$ by

$$
\frac{P_{i}}{Q_{i}}=\frac{1}{a_{1}}+\frac{1}{a_{2}}+\cdots+\frac{1}{a_{i}},
$$

and set $C=Q_{k-1}+P_{k}+c Q_{k}$.

If $k$ is even, set

$$
a=\frac{C-\sqrt{C^{2}+4}}{2}, \quad b=\frac{C+\sqrt{C^{2}+4}}{2} .
$$

Then

$$
\begin{aligned}
& {\left[0 ; \overline{a_{1}, \ldots, a_{k}, c+d m^{n}}\right]_{n=1}^{\infty}} \\
& =\frac{P_{k}}{Q_{k}}+\frac{1}{Q_{k}\left(m d Q_{k}+a\right)} \frac{\sum_{n=0}^{\infty} \frac{\left(b / d Q_{k}\right)^{n} m^{-n(n+3) / 2}}{\sum_{n=0}^{\infty} \frac{\left(b / d Q_{k}\right)^{n} m^{-n(n+1) / 2}}{(1 / m ; 1 / m)_{n}\left(-a / d Q_{k} m ; 1 / m\right)_{n}}}}{(1 / m)_{n}\left(-a / d Q_{k} m^{2} ; 1 / m\right)_{n}}
\end{aligned}
$$

If $k$ is odd, set

$$
a=\frac{C-\sqrt{C^{2}-4}}{2}, \quad b=\frac{C+\sqrt{C^{2}-4}}{2} .
$$

Then

$$
\begin{aligned}
& {\left[0 ; \overline{a_{1}, \ldots, a_{k}, c+d m^{n}}\right]_{n=1}^{\infty}} \\
& =\frac{P_{k}}{Q_{k}}+\frac{-1}{Q_{k}\left(m d Q_{k}+a\right)} \frac{\sum_{n=0}^{\infty} \frac{\left(b / d Q_{k}\right)^{n} m^{-n(n+3) / 2}}{\sum_{n=0}^{\infty} \frac{\left(b / d Q_{k}\right)^{n} m^{-n(n+1) / 2}}{(1 / m ; 1 / m)_{n}\left(-a / d Q_{k} m ; 1 / m\right)_{n}}}}{(1 / m)_{n}\left(-a / d Q_{k} m^{2} ; 1 / m\right)_{n}}
\end{aligned}
$$

Proof. Set $e=c$ in Theorem 5 .

COROLlary 4. Let $c$ and $m$ be integers, $m>1$, and let $d$ be a positive rational such that $d m \in \mathbb{N}$ and $c+d m>0$. Let $k$ be an even positive integer, 
let $F_{i}$ denote the ith Fibonacci number and let $C=2 F_{k}+c F_{k+1}$. Set

$$
a=\frac{C-\sqrt{C^{2}+4}}{2}, \quad b=\frac{C+\sqrt{C^{2}+4}}{2} .
$$

Then

$$
[0 ; \underbrace{\overline{1, \ldots, 1}, c+d m^{n}}_{k}]_{n=1}^{\infty}
$$

$$
=\frac{F_{k}}{F_{k+1}}+\frac{1}{F_{k+1}\left(m d F_{k+1}+a\right)} \frac{\sum_{n=0}^{\infty} \frac{\left(b / d F_{k+1}\right)^{n} m^{-n(n+3) / 2}}{(1 / m ; 1 / m)_{n}\left(-a / d F_{k+1} m^{2} ; 1 / m\right)_{n}}}{\sum_{n=0}^{\infty} \frac{\left(b / d F_{k+1}\right)^{n} m^{-n(n+1) / 2}}{(1 / m ; 1 / m)_{n}\left(-a / d F_{k+1} m ; 1 / m\right)_{n}}} .
$$

Proof. This follows immediately from Corollary 3, upon noting that

$$
[0 ; \underbrace{1, \ldots, 1}_{i}]=\frac{F_{i}}{F_{i+1}} .
$$

We also require some preliminary results before our next construction (see also (2.6) above). The following theorem can be found in [15, p. 85].

TheOREM 6. The canonical contraction of $b_{0}+K_{n=1}^{\infty} a_{n} / b_{n}$ with $C_{0}=$ $A_{1} / B_{1}$

$$
C_{k}=A_{2 k+1}, \quad D_{k}=B_{2 k+1} \quad \text { for } k=1,2, \ldots
$$

exists if and only if $b_{2 k+1} \neq 0$ for $k=0,1,2, \ldots$, and in this case is given by

$$
\begin{aligned}
\frac{b_{0} b_{1}+a_{1}}{b_{1}}- & \frac{a_{1} a_{2} b_{3} / b_{1}}{b_{1}\left(a_{3}+b_{2} b_{3}\right)+a_{2} b_{3}}-\frac{a_{3} a_{4} b_{5} b_{1} / b_{3}}{a_{5}+b_{4} b_{5}+a_{4} b_{5} / b_{3}} \\
& -\frac{a_{5} a_{6} b_{7} / b_{5}}{a_{7}+b_{6} b_{7}+a_{6} b_{7} / b_{5}}-\frac{a_{7} a_{8} b_{9} / b_{7}}{a_{9}+b_{8} b_{9}+a_{8} b_{9} / b_{7}}+\cdots
\end{aligned}
$$

The continued fraction (2.16) is called the odd part of $b_{0}+K_{n=1}^{\infty} a_{n} / b_{n}$. The following corollary is easily deduced from Theorem 6 .

COROLlary 5. The odd part of the continued fraction

$$
\frac{c_{1}}{1}-\frac{c_{2}}{1}+\frac{c_{2}}{1}-\frac{c_{3}}{1}+\frac{c_{3}}{1}-\frac{c_{4}}{1}+\frac{c_{4}}{1}-\cdots
$$

$i s$

$$
c_{1}+\frac{c_{1} c_{2}}{1}+\frac{c_{2} c_{3}}{1}+\frac{c_{3} c_{4}}{1}+\cdots \cdot
$$

This corollary implies the following result. 
Corollary 6. Let $p$ and $a_{i}, i \geq 1$, be complex numbers. If the continued fraction

$$
\begin{aligned}
\frac{1 / p}{1}-\frac{p / a_{1}}{1}+ & \frac{p / a_{1}}{1}-\frac{1 / p a_{2}}{1}+\frac{1 / p a_{2}}{1} \\
& -\frac{p / a_{3}}{1}+\frac{p / a_{3}}{1}-\frac{1 / p a_{4}}{1}+\frac{1 / p a_{4}}{1}-\cdots \\
& -\frac{p / a_{2 n-1}}{1}+\frac{p / a_{2 n-1}}{1}-\frac{1 / p a_{2 n}}{1}+\frac{1 / p a_{2 n}}{1}-\cdots
\end{aligned}
$$

converges, then

$$
\left[0 ; \overline{p, \frac{-a_{2 n-1}}{p^{2}},-p, a_{2 n}}\right]_{n=1}^{\infty}=\frac{1}{p}+\left[0 ; a_{1}, a_{2}, a_{3}, \ldots\right] .
$$

Proof. The continued fraction at (2.17) is easily seen to be equivalent to the continued fraction on the left side of (2.18), after a sequence of similarity transformations is applied to the former continued fraction to transform all the partial numerators into " 1 "'s. On the other hand, since the continued fraction at (2.17) converges, it is equal to its odd part, which, by Corollary 5 , is the continued fraction

$$
\begin{aligned}
\frac{1}{p}+\frac{1 / a_{1}}{1}+\frac{1 / a_{1} a_{2}}{1}+\frac{1 / a_{2} a_{3}}{1}+\frac{1 / a_{3} a_{4}}{1}+ & \cdots \\
& =\frac{1}{p}+\left[0 ; a_{1}, a_{2}, a_{3}, \ldots\right] .
\end{aligned}
$$

We will also make use of Worpitzky's theorem (see [15, pp. 35-36]) to ensure convergence of the continued fraction at (2.17).

THEOREM 7 (Worpitzky). Let the continued fraction $K_{n=1}^{\infty} a_{n} / 1$ be such that $\left|a_{n}\right| \leq 1 / 4$ for $n \geq 1$. Then $K_{n=1}^{\infty} a_{n} / 1$ converges. All approximants of the continued fraction lie in the disk $|w|<1 / 2$ and the value of the continued fraction is in the disk $|w| \leq 1 / 2$.

Corollary 6 can now be used to derive the limit of new Tasoevian continued fractions from existing Tasoevian continued fractions whose values are known. The new continued fraction will contain an additional free parameter. We give two examples.

Theorem 8. Let $c, e, m$ and $p$ be integers, with $m>1$ and $p>1$. Let $d$ be a positive rational such that $d m, d c m / e \in \mathbb{N}$, and $c+d c m / e-1, e+$ $d m^{2}-1>0$. Set

$$
a=\frac{e-\sqrt{e^{2}+4 e / c p^{2}}}{2}, \quad b=\frac{e+\sqrt{e^{2}+4 e / c p^{2}}}{2} .
$$


Then

$$
\begin{aligned}
& {\left[0 ; \overline{p-1,1, c+\frac{d c}{e} m^{2 n-1}-1, p-1,1, e+d m^{2 n}-1}\right]_{n=1}^{\infty}}
\end{aligned}
$$

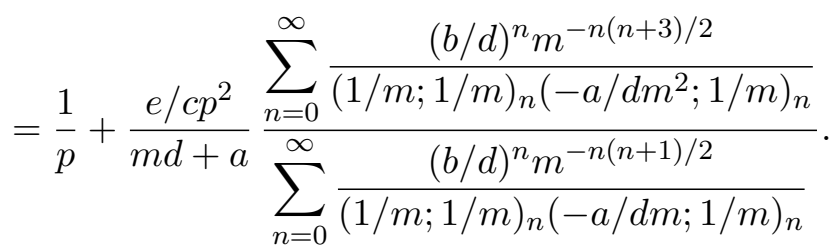

Proof. Replace $c$ by $c p^{2}$ in Theorem 2 and let the resulting continued fraction be the continued fraction on the right side of (2.18). After the negatives are removed (see the remark before Corollary 2) from the corresponding continued fraction on the left side of (2.18), the continued fraction on the left side at (2.19) is produced and the result follows.

TheOREM 9. Let $u$ and $v$ be positive integers, $u, v>1$, and let $e$ and $f$ be rationals such that $e u-1, f v-1 \in \mathbb{N}$. Then

$$
\left[0 ; \overline{p-1,1, e u^{n}-1, p-1,1, f v^{n}-1}\right]_{n=1}^{\infty}
$$

$$
=\frac{1}{p}+\left(\frac{1}{e p^{2} u}-\frac{1}{e^{2} f p^{4} u^{2} v+e p^{2}}\right) \frac{\sum_{n=0}^{\infty} \frac{\left(e f p^{2}\right)^{-n}(u v)^{-n(n+3) / 2}}{(1 / u v ; 1 / u v)_{n}\left(-1 / e f p^{2} u^{3} v^{2} ; 1 / u v\right)_{n}}}{\sum_{n=0}^{\infty} \frac{\left(e f p^{2}\right)^{-n}(u v)^{-n(n+1) / 2}}{(1 / u v ; 1 / u v)_{n}\left(-1 / e f p^{2} u^{2} v ; 1 / u v\right)_{n}}} .
$$

Proof. The proof is similar to that of Theorem 8, except we replace $e$ with $e p^{2}$ in Theorem 4.

REMARK. It is clear that many other continued fractions of Tasoevian type could be produced from those listed in this section, by either replacing various parameters by their negatives, or applying Corollary 6 differently (for example, by replacing $m$ by $m p$ or $m p^{2}$ (instead of replacing $e$ by $e p^{2}$ ) in Theorems 8 and 9). However, we feel these methods have been sufficiently illustrated here and refrain from further examples.

2.1. Some implications of a result of Hurwitz and Châtelet. Some of the numbers whose Hurwitzian or Tasoevian continued fraction expansions are described in this paper are of the form

$$
\beta=\frac{r \alpha+t}{s},
$$

where $\alpha$ is a number with a known continued fraction expansion, and $r$, $s$ and $t$ are integers. Sometime after completing an earlier version of this paper, I became aware of two recent papers by Takao Komatsu $[10,11]$ that considered some similar types Hurwitzian and Tasoevian continued 
fractions. Here I will describe a framework that explains the derivation of many of those, and also several of the continued fractions in the present paper.

Komatsu's results derive from applications of the following lemma, which he says is essentially due to Hurwitz and Châtelet.

LEMMA 1. Let $\left[a_{0} ; a_{1}, a_{2}, \ldots\right]$ be the regular continued fraction of the irrational number $\alpha$ and denote its nth convergent by $p_{n} / q_{n}=\left[a_{0} ; a_{1}, \ldots, a_{n}\right]$. Moreover, let $\beta=\left(r_{0} \alpha+t_{0}\right) / s_{0}$, where $r_{0}, s_{0}$ and $t_{0}$ are integers with $r_{0}>0$, $s_{0}>0$ and $r_{0} s_{0}=N>1$. For an arbitrary index $\nu \geq 1$ we have

$$
\frac{r_{0}\left[a_{0} ; a_{1}, \ldots, a_{\nu-1}\right]+t_{0}}{s_{0}}=\frac{r_{0} p_{\nu-1}+t_{0} q_{\nu-1}}{s_{0} q_{\nu-1}}=\left[b_{0} ; b_{1}, \ldots, b_{\mu-1}\right],
$$

where the index $\mu$ is adjusted so that $\mu \equiv \nu(\bmod 2)$. Denote its convergent by

$$
\frac{p_{\mu-1}^{\prime}}{q_{\mu-1}}=\left[b_{0} ; b_{1}, b_{2}, \ldots, b_{\mu-1}\right] .
$$

Then three integers $t_{1}, r_{1}$ and $s_{1}$ are uniquely given satisfying the matrix formula

$$
\left(\begin{array}{cc}
r_{0} & t_{0} \\
0 & s_{0}
\end{array}\right)\left(\begin{array}{ll}
p_{\nu-1} & p_{\nu-2} \\
q_{\nu-1} & q_{\nu-2}
\end{array}\right)=\left(\begin{array}{ll}
p_{\mu-1}^{\prime} & p_{\mu-2}^{\prime} \\
q_{\mu-1}^{\prime} & q_{\mu-2}^{\prime}
\end{array}\right)\left(\begin{array}{cc}
r_{1} & t_{1} \\
0 & s_{1}
\end{array}\right),
$$

where $r_{1}>0, s_{1}>0, r_{1} s_{1}=N,-s_{1} \leq t_{1} \leq r_{1}$ and $\beta=\left[b_{0} ; b_{1}, \ldots, b_{\mu-1}, \beta_{\mu}\right]$ with $\beta_{\mu}=\left(r_{1} \alpha_{\nu}+t_{1}\right) / s_{1}$.

For ease of notation in what follows, let

$$
A_{i}:=\left(\begin{array}{cc}
a_{i} & 1 \\
1 & 0
\end{array}\right), \quad B_{i}:=\left(\begin{array}{cc}
b_{i} & 1 \\
1 & 0
\end{array}\right), \quad R_{i}:=\left(\begin{array}{cc}
r_{i} & t_{i} \\
0 & s_{i}
\end{array}\right) .
$$

The above lemma implies that if there exist integers $j, k$ and $m$, and sets of integers $r_{i}, s_{i}$ and $t_{i}, 0 \leq i \leq m-1$, such that for all integers $n \geq 0$,

$$
\begin{aligned}
R_{0} A_{n j} A_{n j+1} \ldots A_{n j+i_{1}-1} & =B_{n k} B_{n k+1} \ldots B_{n k+j_{1}-1} R_{1}, \\
R_{1} A_{n j+i_{1}} A_{n j+i_{1}+1} \ldots A_{n j+i_{2}-1} & =B_{n k+j_{1}} B_{n k+j_{1}+1} \ldots B_{n k+j_{2}-1} R_{2}, \\
R_{2} A_{n j+i_{2}} A_{n j+i_{2}+1} \ldots A_{n j+i_{3}-1} & =B_{n k+j_{2}} B_{n k+j_{2}+1} \ldots B_{n k+j_{3}-1} R_{3}, \\
& \vdots \\
R_{m-2} A_{n j+i_{m-2}} \ldots A_{n j+i_{m-1}-1} & =B_{n k+j_{m-2}} \ldots B_{n k+j_{m-1}-1} R_{m-1}, \\
R_{m-1} A_{n j+i_{m-1}} \ldots A_{n j+j-1} & =B_{n k+j_{m-1}} \ldots B_{n k+k-1} R_{0},
\end{aligned}
$$

then

$$
\frac{r_{0} \alpha+t_{0}}{s_{0}}=\left[b_{0} ; b_{1}, b_{2}, \ldots\right]
$$


More concisely, it implies that if there exist integers $j$ and $k$ such that for all integers $n \geq 0$,

$$
R_{0} A_{n j} A_{n j+1} \ldots A_{n j+j-1}=B_{n k} B_{n k+1} \ldots B_{n k+k-1} R_{0},
$$

then

$$
\frac{r_{0} \alpha+t_{0}}{s_{0}}=\left[b_{0} ; b_{1}, b_{2}, \ldots\right]
$$

Theorem 1 in [10] is a consequence of the fact that

$$
\begin{aligned}
\left(\begin{array}{cc}
v & -1 \\
0 & v
\end{array}\right)\left(\begin{array}{cc}
a_{2 j} & 1 \\
1 & 0
\end{array}\right) & \left(\begin{array}{cc}
a_{2 j+1} & 1 \\
1 & 0
\end{array}\right) \\
= & \left(\begin{array}{cc}
a_{2 j}-1 & 1 \\
1 & 0
\end{array}\right)\left(\begin{array}{ll}
1 & 1 \\
1 & 0
\end{array}\right)\left(\begin{array}{cc}
v-1 & 1 \\
1 & 0
\end{array}\right) \\
& \times\left(\begin{array}{cc}
\frac{a_{2 j+1}}{v^{2}}-1 & 1 \\
1 & 0
\end{array}\right)\left(\begin{array}{ll}
1 & 1 \\
1 & 0
\end{array}\right)\left(\begin{array}{cc}
v-1 & 1 \\
1 & 0
\end{array}\right)\left(\begin{array}{cc}
v & -1 \\
0 & v
\end{array}\right) .
\end{aligned}
$$

Similarly, Theorem 3 in [10] essentially follows from the fact that

$$
\begin{aligned}
\left(\begin{array}{cc}
1 & v-v^{2} \\
0 & v^{2}
\end{array}\right) & \left(\begin{array}{cc}
a_{2 j} & 1 \\
1 & 0
\end{array}\right)\left(\begin{array}{cc}
a_{2 j+1} & 1 \\
1 & 0
\end{array}\right) \\
= & \left(\begin{array}{cc}
\frac{a_{2 j}}{v^{2}}-1 & 1 \\
1 & 0
\end{array}\right)\left(\begin{array}{cc}
v-1 & 1 \\
1 & 0
\end{array}\right)\left(\begin{array}{ll}
1 & 1 \\
1 & 0
\end{array}\right) \\
& \times\left(\begin{array}{cc}
a_{2 j+1}-1 & 1 \\
1 & 0
\end{array}\right)\left(\begin{array}{cc}
v-1 & 1 \\
1 & 0
\end{array}\right)\left(\begin{array}{ll}
1 & 1 \\
1 & 0
\end{array}\right)\left(\begin{array}{cc}
1 & v-v^{2} \\
0 & v^{2}
\end{array}\right),
\end{aligned}
$$

and Theorems $7-10$ in [10] derives from the fact that

$$
\left(\begin{array}{cc}
1 & -1 \\
0 & v
\end{array}\right)\left(\begin{array}{cc}
a_{j} & 1 \\
1 & 0
\end{array}\right)=\left(\begin{array}{cc}
\frac{a_{j}}{v}-1 & 1 \\
1 & 0
\end{array}\right)\left(\begin{array}{ll}
1 & 1 \\
1 & 0
\end{array}\right)\left(\begin{array}{cc}
v-1 & 1 \\
1 & 0
\end{array}\right)\left(\begin{array}{cc}
1 & -1 \\
0 & v
\end{array}\right)
$$

after setting $v=2$. Theorem 1 in [11] follows from the fact that

$$
\begin{aligned}
\left(\begin{array}{cc}
v & -l \\
0 & v
\end{array}\right) & \left(\begin{array}{cc}
a_{2 j} & 1 \\
1 & 0
\end{array}\right)\left(\begin{array}{cc}
a_{2 j+1} & 1 \\
1 & 0
\end{array}\right) \\
= & \left(\begin{array}{cc}
a_{2 j}-1 & 1 \\
1 & 0
\end{array}\right)\left(\begin{array}{ll}
1 & 1 \\
1 & 0
\end{array}\right)\left(\begin{array}{rl}
\frac{v-l-1}{l} & 1 \\
1 & 0
\end{array}\right)\left(\begin{array}{cc}
l-1 & 1 \\
1 & 0
\end{array}\right) \\
& \times\left(\begin{array}{ll}
1 & 1 \\
1 & 0
\end{array}\right)\left(\begin{array}{cc}
\frac{a_{2 j+1}}{v^{2}}-1 & 1 \\
1 & 0
\end{array}\right)\left(\begin{array}{ll}
l & 1 \\
1 & 0
\end{array}\right)\left(\begin{array}{cc}
\frac{v-1}{l} & 1 \\
1 & 0
\end{array}\right)\left(\begin{array}{cc}
v & -l \\
0 & v
\end{array}\right)
\end{aligned}
$$




$$
\begin{aligned}
= & \left(\begin{array}{cc}
a_{2 j}-1 & 1 \\
1 & 0
\end{array}\right)\left(\begin{array}{ll}
1 & 1 \\
1 & 0
\end{array}\right)\left(\begin{array}{cc}
\frac{v-2 l+1}{l} & 1 \\
1 & 0
\end{array}\right)\left(\begin{array}{ll}
1 & 1 \\
1 & 0
\end{array}\right)\left(\begin{array}{cc}
l-1 & 1 \\
1 & 0
\end{array}\right) \\
& \times\left(\begin{array}{cc}
\frac{a_{2 j+1}}{v^{2}}-1 & 1 \\
1 & 0
\end{array}\right)\left(\begin{array}{ll}
1 & 1 \\
1 & 0
\end{array}\right)\left(\begin{array}{cc}
l-2 & 1 \\
1 & 0
\end{array}\right)\left(\begin{array}{ll}
1 & 1 \\
1 & 0
\end{array}\right)\left(\begin{array}{cc}
\frac{v-l+1}{l} & 1 \\
1 & 0
\end{array}\right)\left(\begin{array}{cc}
v & -l \\
0 & v
\end{array}\right) .
\end{aligned}
$$

Many other Hurwitzian and Tasoevian continued fraction expansions, including several of the results in [11], may be derived from existing continued fraction expansions using the matrix identities above, and other similar such matrix identities, but we leave it to the reader to derive the specific matrix identities corresponding to particular theorems.

In the present paper, the continued fraction identity at (2.18) may be regarded as following from a special case $\left(m=p^{2}, a_{0}=0\right)$ of the matrix identity

$$
\begin{aligned}
\left(\begin{array}{cc}
m & p \\
0 & p^{2}
\end{array}\right)\left(\begin{array}{cc}
a_{2 j} & 1 \\
1 & 0
\end{array}\right) & \left(\begin{array}{cc}
a_{2 j+1} & 1 \\
1 & 0
\end{array}\right) \\
= & \left(\begin{array}{cc}
\frac{m a_{2 j}}{p^{2}} & 1 \\
1 & 0
\end{array}\right)\left(\begin{array}{cc}
p & 1 \\
1 & 0
\end{array}\right)\left(\begin{array}{cc}
\frac{-a_{2 j+1}}{m} & 1 \\
1 & 0
\end{array}\right)\left(\begin{array}{cc}
-p & 1 \\
1 & 0
\end{array}\right)\left(\begin{array}{cc}
m & p \\
0 & p^{2}
\end{array}\right) .
\end{aligned}
$$

However we keep the existing proof to manifest the variety of ways of deriving these continued fraction identities. Also, this particular identity was not the basis for any of the results of Komatsu [10,11] that we referred to, and so the continued fractions we derive by using it do not follow from the theorems in these papers.

We do not consider any further in the present paper the kind of matrix identities exhibited above, although it should be obvious that similar matrix identities will give rise to many other families of Hurwitzian and Tasoevian continued fractions.

3. Hurwitzian continued fractions. We first recall some of the wellknown classes of Hurwitzian continued fractions and consider some elementary generalizations of them. We first note that Lehmer's continued fraction (1.2),

$$
[0 ; a, a+b, a+2 b, a+3 b, \ldots]=\frac{I_{(a / b)}(2 / b)}{I_{(a / b)-1}(2 / b)},
$$

can easily be generalized. Replace $a$ by $a \sqrt{u v}$ and $b$ by $b \sqrt{u v}$, multiply both sides of (1.2) by $\sqrt{v / u}$, apply a sequence of similarity transformations to the resulting continued fraction to make it regular once more, and we get 


$$
[0 ; u a, v(a+b), u(a+2 b), v(a+3 b), \ldots]=\sqrt{\frac{v}{u}} \frac{I_{(a / b)}(2 / b \sqrt{u v})}{I_{(a / b)-1}(2 / b \sqrt{u v})} .
$$

Komatsu also derives this generalization in [5], but his derivation is more complicated. We note that several of the well-known classes of Hurwitzian continued fractions follow as special cases of (3.1). For example, before removing the negative partial quotients, Lambert's continued fraction (1.1) gives

$$
\sqrt{\frac{v}{u}} \tan \frac{1}{\sqrt{u v}}=[0 ; u,-3 v, 5 u,-7 v, \ldots],
$$

which follows upon setting $a=1, b=2$ and replacing $v$ with $-v$. The continued fraction

$$
\sqrt{\frac{v}{u}} \tanh \frac{1}{\sqrt{u v}}=[0 ; u, 3 v, 5 u, 7 v, \ldots]
$$

is clearly also a special case. The continued fraction

$$
[0 ; \overline{(4 n+2) s}]_{n=0}^{\infty}=\frac{e^{1 / s}-1}{e^{1 / s}+1}
$$

is clearly a special case of (1.2). Thus, as Komatsu indicated in [9], the continued fraction at (3.1) may be used to generalize several of the wellknown Hurwitzian continued fraction expansions. As in Corollary 2, further variations follow upon replacing some of the parameters by their negatives.

We also recall a well-known continued fraction expansion for $e^{z}, z \in \mathbb{C}$ (see [15, p. 563], for example):

$$
e^{z}=\frac{1}{1}-\frac{z}{1}+\frac{z}{2}-\frac{z}{3}+\frac{z}{2}-\frac{z}{5}+\frac{z}{2}-\frac{z}{7}+\cdots .
$$

Set $z=1 / m^{2}$ and apply a sequence of similarity transformations to the resulting continued fraction to get

$$
\begin{aligned}
m\left(1-e^{-1 / m^{2}}\right) & =[0 ; \overline{(4 n+1) m, 2 m,-(4 n+3) m,-2 m}]_{n=0}^{\infty} \\
& =[0 ; m, \overline{2 m-1,1,(2 n+1) m-1}]_{n=1}^{\infty} .
\end{aligned}
$$

If we set $m=\sqrt{u v}$ and multiply the left side of (3.5) and the first continued fraction on the right side of (3.5) by $\sqrt{v / u}$, we get

$$
\begin{aligned}
v\left(1-e^{-1 / u v}\right) & =[0 ; \overline{(4 n+1) u, 2 v,-(4 n+3) u,-2 v}]_{n=0}^{\infty} \\
& =[0 ; u, \overline{2 v-1,1,(2 n+1) u-1}]_{n=1}^{\infty} .
\end{aligned}
$$

We have not seen the continued fraction expansions at (3.5) and (3.6) elsewhere.

We are now ready to derive several new families of Hurwitzian continued fractions, using Corollary 6. 
TheOREM 10. Let $a, b, p, u$ and $v$ be integers restricted in the case of each continued fraction below so that the partial quotients are all positive. Then

$$
\begin{aligned}
& {[0 ; \overline{p-1,1, u(a+2 n b)-1, p-1,1, v(a+(2 n+1) b)-1}]_{n=0}^{\infty}} \\
& =\frac{1}{p}+\frac{1}{p} \sqrt{\frac{v}{u}} \frac{I_{(a / b)}(2 / b p \sqrt{u v})}{I_{(a / b)-1}(2 / b p \sqrt{u v})}, \\
& {[0 ; p-1, \overline{1,(4 n+1) u-1, p,(4 n+3) v-1,1, p-2}]_{n=0}^{\infty}} \\
& =\frac{1}{p}+\frac{1}{p} \sqrt{\frac{v}{u}} \tan \frac{1}{p \sqrt{u v}}, \\
& {[0 ; \overline{p-1,1,(4 n+1) u-1, p-1,1,(4 n+3) v-1}]_{n=0}^{\infty}} \\
& =\frac{1}{p}+\frac{1}{p} \sqrt{\frac{v}{u}} \tanh \frac{1}{p \sqrt{u v}}, \\
& {[0 ; p-1, \overline{1,(4 n+1) u-1, p-1,1,2 v-1, p,(4 n+3) u-1},} \\
& \overline{1, p-1,2 v-1,1, p-2}]_{n=0}^{\infty} \\
& =\frac{1}{p}+v\left(1-e^{-1 / u p^{2} v}\right) \text {. }
\end{aligned}
$$

Proof. The claimed identities follow by applying the result in Corollary 6 to, in turn, (3.1), (3.2), (3.3) and (3.6) (replace $u$ by $u p^{2}$ in each case), and then removing the negative signs from the resulting continued fractions.

REMARK. Variants of each of these continued fraction identities could be produced by replacing some of the parameters in each expansion in Theorem 10 by their negatives, as in Corollary 2, but we do not consider that here.

3.1. Finite continued fractions containing arithmetic progressions. Here we find expressions for finite continued fractions of the form $[0 ; a, a+b$, $a+2 b, a+3 b, \ldots, a+(n-1) b]$ and $[0 ; a, c, a+b, c+d, a+2 b, c+2 d, \ldots$, $a+(n-1) b, c+(n-1) d]$, where $a, b, c$ and $d$ satisfy a simple algebraic relation.

We first prove the following theorem.

TheOREM 11. Let

$$
\frac{P_{n}}{Q_{n}}:=\frac{-c}{a}-\frac{c}{a+b}-\frac{c}{a+2 b}-\cdots-\frac{c}{a+(n-1) b}
$$

denote the nth approximant of the continued fraction $K_{j=0}^{\infty}-c /(a+j b)$. 
Then

$$
\begin{aligned}
P_{n} & =\sum_{i=1}^{\lfloor(n+1) / 2\rfloor}\left(\begin{array}{c}
n-i \\
i-1
\end{array}\right)(-c)^{i} \prod_{j=i}^{n-i}(a+j b), \\
Q_{n} & =\sum_{i=0}^{\lfloor n / 2\rfloor}\left(\begin{array}{c}
n-i \\
i
\end{array}\right)(-c)^{i} \prod_{j=i}^{n-1-i}(a+j b) .
\end{aligned}
$$

Proof. The statements are easily checked to be true for $n=1$ and $n=2$ (as usual, the empty product is taken to be equal to 1). Now suppose the statements are true for $n=1, \ldots, k$. We compute

$$
\begin{aligned}
P_{k+1}= & (a+k b) P_{k}-c P_{k-1} \\
= & (a+k b) \sum_{i=1}^{\lfloor(k+1) / 2\rfloor}\left(\begin{array}{c}
k-i \\
i-1
\end{array}\right)(-c)^{i} \prod_{j=i}^{k-i}(a+j b) \\
& -c \sum_{i=1}^{\lfloor k / 2\rfloor}\left(\begin{array}{c}
k-1-i \\
i-1
\end{array}\right)(-c)^{i} \prod_{j=i}^{k-1-i}(a+j b) \\
= & -c \prod_{j=1}^{k}(a+j b)+(a+k b) \sum_{i=2}^{\lfloor(k+1) / 2\rfloor}\left(\begin{array}{c}
k-i \\
i-1
\end{array}\right)(-c)^{i} \prod_{j=i}^{k-i}(a+j b) \\
& +\sum_{i=2}^{\lfloor k / 2\rfloor+1}\left(\begin{array}{c}
k-i \\
i-2
\end{array}\right)(-c)^{i} \prod_{j=i-1}^{k-i}(a+j b) .
\end{aligned}
$$

If $k$ is odd, then

$$
\lfloor(k+1) / 2\rfloor=\lfloor k / 2\rfloor+1=\lfloor(k+2) / 2\rfloor
$$

and

$$
\begin{aligned}
(a+k b) & \left(\begin{array}{c}
k-i \\
i-1
\end{array}\right)(-c)^{i} \prod_{j=i}^{k-i}(a+j b)+\left(\begin{array}{c}
k-i \\
i-2
\end{array}\right)(-c)^{i} \prod_{j=i-1}^{k-i}(a+j b) \\
= & (-c)^{i} \prod_{j=i}^{k-i}(a+j b) \frac{(k-i) !}{(i-2) !(k-2 i+1) !}\left(\frac{a+k b}{i-1}+\frac{a+(i-1) b}{k-2 i+2}\right) \\
= & (-c)^{i} \prod_{j=i}^{k-i}(a+j b) \frac{(k-i) !}{(i-2) !(k-2 i+1) !} \frac{(k-i+1)(a+(k-i+1) b)}{(i-1)(k-2 i+2)} \\
= & \left(\begin{array}{c}
k+1-i \\
i-1
\end{array}\right)(-c)^{i} \prod_{j=i}^{k+1-i}(a+j b),
\end{aligned}
$$


and hence

$$
P_{k+1}=\sum_{i=1}^{\lfloor(k+2) / 2\rfloor}\left(\begin{array}{c}
k+1-i \\
i-1
\end{array}\right)(-c)^{i} \prod_{j=i}^{k+1-i}(a+j b) .
$$

If $k$ is even, the extra $\lfloor k / 2\rfloor+1$-th term at (3.13) provides the $\lfloor(k+2) / 2\rfloor$-th term in the sum above. The proof of (3.12) for $P_{n}$ now follows.

The proof for $Q_{n}$ is virtually identical, and so is omitted.

COROLlary 7 . Let $a$ and $b$ be positive integers. Then

$$
\frac{1}{a}+\frac{1}{a+b}+\cdots+\frac{1}{a+(n-1) b}=\frac{\sum_{i=1}^{\lfloor(n+1) / 2\rfloor}\left(\begin{array}{c}
n-i \\
i-1
\end{array}\right) \prod_{j=i}^{n-i}(a+j b)}{\sum_{i=0}^{\lfloor n / 2\rfloor}\left(\begin{array}{c}
n-i \\
i
\end{array}\right) \prod_{j=i}^{n-1-i}(a+j b)} .
$$

Let $f, g, h$ and $k$ be integers such that $2 g h=k(2 f+h)$. Then

$$
\begin{aligned}
\frac{1}{f}+\frac{1}{g}+\frac{1}{f+h}+ & \frac{1}{g+k}+\cdots+\frac{1}{f+(n-1) h}+\frac{1}{g+(n-1) k} \\
= & \frac{\sum_{i=1}^{n}\left(\begin{array}{c}
2 n-i \\
i-1
\end{array}\right)\left(\frac{2 g}{2 f+h}\right)^{2 n-i} \prod_{j=i}^{2 n-i}\left(f+j \frac{h}{2}\right)}{\sum_{i=0}^{n}\left(\begin{array}{c}
2 n-i \\
i
\end{array}\right)\left(\frac{2 g}{2 f+h}\right)^{2 n-1-i} \prod_{j=i}^{2 n-1-i}\left(f+j \frac{h}{2}\right)} .
\end{aligned}
$$

Proof. The identity at (3.14) follows immediately, upon setting $c=-1$ in Theorem 11. For (3.15), it is easy to see that

$$
\begin{aligned}
\frac{-c}{a}-\frac{c}{a+b}-\frac{c}{a+2 b}- & \cdots-\frac{c}{a+(2 n-1) b} \\
= & \frac{1}{-a / c}+\frac{1}{a+b}+\frac{1}{-a / c-2 b / c}+\frac{1}{a+3 b}+\cdots \\
& +\frac{1}{-a / c-(2 n-2) b / c}+\frac{1}{a+(2 n-1) b} .
\end{aligned}
$$

Now make the substitutions

$$
a=\frac{2 f g}{2 f+h}, \quad b=\frac{g h}{2 f+h}, \quad c=-\frac{2 g}{2 f+h},
$$

and the continued fraction at (3.15) is produced. The result follows, after some simple manipulations, upon making the same substitutions into the ratio $P_{2 n} / Q_{2 n}$, where $P_{2 n}$ and $Q_{2 n}$ are as defined at (3.12). 
Lehmer's result (1.2) easily follows from (3.14), upon re-indexing the numerator on the right side by replacing $i$ with $i+1$, dividing top and bottom on the right side by $\prod_{j=0}^{n-1}(a+j b)$, performing some simple algebraic manipulations, and then letting $n \rightarrow \infty$.

Corollary 8 (Lehmer [14]). Let $a$ and $b$ be positive integers. Then

$$
[0 ; a, a+b, a+2 b, a+3 b, \ldots]=\frac{1}{b} \frac{\sum_{k=0}^{\infty} \frac{b^{-2 k}}{(a / b)_{k+1} k !}}{\sum_{k=0}^{\infty} \frac{b^{-2 k}}{(a / b)_{k} k !}} .
$$

\section{Hurwitzian and Tasoevian continued fractions with arbitrar-} ily long quasi-period. We conclude by noting that the construction described in Corollary 6 can be iterated to produce both Hurwitzian and Tasoevian continued fractions with arbitrarily long quasi-period, with arbitrarily many free parameters and whose limits can be determined. We give one example, with seven free parameters and quasi-period of length 24 , to illustrate this.

Theorem 12. Let e, $f, p>1, q>1, r>2, u>1$ and $v>1$ be positive integers. Let $E=e p^{2} q^{4} r^{8}$. Then

$$
\begin{aligned}
& {\left[0 ; \overline{r-1,1, q-1, r, p-1,1, r-1, q-1,1, r-1, e u^{n}-1,1},\right.} \\
& \left.\quad \frac{1}{r-2,1, q-1, r-1,1, p-1, r, q-1,1, r-2,1, f v^{n}-1}\right]_{n=1}^{\infty} \\
& =\frac{1}{p q^{2} r^{4}}+\frac{1}{q r^{2}}+\frac{1}{r} \\
& \quad+\left(\frac{1}{E u}-\frac{1}{E^{2} f u^{2} v+E}\right) \frac{\sum_{n=0}^{\infty} \frac{(E f)^{-n}(u v)^{-n(n+3) / 2}}{\sum_{n=0}^{\infty} \frac{(E f)^{-n}(u v)^{-n(n+1) / 2}}{(1 / u v ; 1 / u v)_{n}\left(-1 / E f u^{3} v^{2} ; 1 / u v\right)_{n}}} .}{(1 / u v ; 1 / u v)_{n}\left(-1 / E f u^{2} v ; 1 / u v\right)_{n}} .
\end{aligned}
$$

Proof. For ease of notation, let

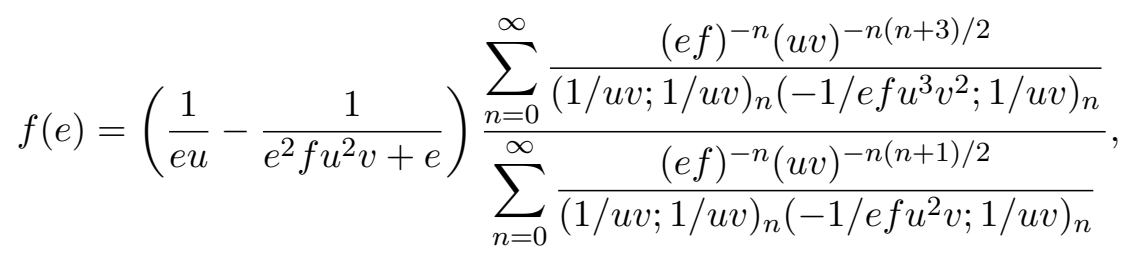

so that, by Theorem 4 ,

$$
\left[0 ; \overline{e u^{n}, f v^{n}}\right]_{n=1}^{\infty}=f(e) .
$$


Replace $e$ with $e p^{2}$ and, by Corollary 6 ,

$$
\left[0 ; \overline{p,-e u^{n},-p, f v^{n}}\right]_{n=1}^{\infty}=\frac{1}{p}+f\left(e p^{2}\right) .
$$

Replace $p$ with $p q^{2}$ and, again by Corollary 6 ,

$$
\left[0 ; \overline{q,-p,-q,-e u^{n}, q, p,-q, f v^{n}}\right]_{n=1}^{\infty}=\frac{1}{q}+\frac{1}{p q^{2}}+f\left(e p^{2} q^{4}\right) .
$$

Repeat this step once more, by replacing $q$ with $q r^{2}$, and then

$$
\begin{aligned}
{\left[0 ; \overline{r,-q,-r,-p, r, q,-r,-e u^{n}, r,-q,-r, p, r, q,-r, f v^{n}}\right]_{n=1}^{\infty} } \\
=\frac{1}{r}+\frac{1}{q r^{2}}+\frac{1}{p q^{2} r^{4}}+f\left(e p^{2} q^{4} r^{8}\right) .
\end{aligned}
$$

Finally, remove the negatives from the continued fraction to get (4.1).

\section{References}

[1] D. Bowman, J. Mc Laughlin and N. J. Wyshinski, A q-continued fraction, Int. J. Number Theory 2 (2006), 523-547.

[2] A. Hurwitz, Über die Kettenbrüche, deren Teilnenner arithmetische Reihen bilden, Vierteljahrsschrift Naturforsch. Ges. Zurich 41 (1896), 2. Teil, 34-64.

[3] —, Über die Kettenbruchentwicklung der Zahl e, in: Math. Werke, Band 2, Birkhäuser, Basel, 1933, 129-133.

[4] T. Komatsu, On Tasoev's continued fractions, Math. Proc. Cambridge Philos. Soc. 134 (2003), 1-12.

[5] -, On Hurwitzian and Tasoev's continued fractions, Acta Arith. 107 (2003), 161177.

[6] -, Tasoev's continued fractions and Rogers-Ramanujan continued fractions, J. Number Theory 109 (2004), 27-40.

[7] —, Hurwitz and Tasoev continued fractions, Monatsh. Math. 145 (2005), 47-60.

[8] -, Hurwitz and Tasoev continued fractions with long period, Math. Pannon. 17 (2006), no. 1, 91-110.

[9] - Continued fraction of $e^{2}$ with confluent hypergeometric functions, Liet. Mat. Rink. 46 (2006), 513-531.

[10] -, Hurwitz continued fractions with confluent hypergeometric functions, Czechoslovak Math. J. 57 (132) (2007), 919-932.

[11] - More on Tasoev and Hurwitz continued fractions, to appear.

[12] J. L. Lambert, Beiträge zum Gebrauche der Mathematik und deren Anwendung, 2 Theil, Verlag der Buchhandlung der Realschule, Berlin, 1770.

[13] D. N. Lehmer, Arithmetical theory of certain Hurwitzian continued fractions, Amer. J. Math. 40 (1918), 375-390.

[14] D. H. Lehmer, Continued fractions containing arithmetic progressions, Scripta Math. 29 (1973), 17-24.

[15] L. Lorentzen and H. Waadeland, Continued Fractions with Applications, Stud. Comput. Math. 3, North-Holland, Amsterdam, 1992.

[16] J. Mc Laughlin and N. J. Wyshinski, Ramanujan and the regular continued fraction expansion of real numbers, Math. Proc. Cambridge Philos. Soc. 138 (2005), 367-381. 
[17] A. J. van der Poorten, Explicit formulas for units in certain quadratic number fields, in: Algorithmic Number Theory (Ithaca, NY, 1994), Lecture Notes in Comput. Sci. 877, Springer, Berlin, 1994, 194-208.

[18] B. G. Tasoev, Certain problems in the theory of continued fractions, Trudy Tbiliss. Univ. Mat. Mekh. Astronom. 16-17 (1984), 53-83 (in Russian).

[19] -, On rational approximations of some numbers, Mat. Zametki 67 (2000), 931-937 (in Russian); English transl.: Math. Notes 67 (2000), 786-791.

Mathematics Department

West Chester University

124 Anderson Hall

West Chester, PA 19383, U.S.A.

E-mail: jmclaughl@wcupa.edu

Received on 13.2.2008

and in revised form on 28.7.2008 